\title{
Technical Note: A generic law-of-the-minimum flux limiter for simulating substrate limitation in biogeochemical models
}

\author{
J. Y. Tang and W. J. Riley \\ Department of Climate and Carbon Sciences, Lawrence Berkeley National Laboratory, Berkeley, CA, USA \\ Correspondence to: J. Y. Tang (jinyuntang@lbl.gov)
}

Received: 20 July 2015 - Published in Biogeosciences Discuss.: 19 August 2015

Revised: 9 December 2015 - Accepted: 22 January 2016 - Published: 5 February 2016

\begin{abstract}
We present a generic flux limiter to account for mass limitations from an arbitrary number of substrates in a biogeochemical reaction network. The flux limiter is based on the observation that substrate (e.g., nitrogen, phosphorus) limitation in biogeochemical models can be represented as to ensure mass conservative and non-negative numerical solutions to the governing ordinary differential equations. Application of the flux limiter includes two steps: (1) formulation of the biogeochemical processes with a matrix of stoichiometric coefficients and (2) application of Liebig's law of the minimum using the dynamic stoichiometric relationship of the reactants. This approach contrasts with the ad hoc down-regulation approaches that are implemented in many existing models (such as CLM4.5 and the ACME (Accelerated Climate Modeling for Energy) Land Model (ALM)) of carbon and nutrient interactions, which are error prone when adding new processes, even for experienced modelers. Through an example implementation with a CENTURYlike decomposition model that includes carbon, nitrogen, and phosphorus, we show that our approach (1) produced almost identical results to that from the ad hoc down-regulation approaches under non-limiting nutrient conditions, (2) properly resolved the negative solutions under substrate-limited conditions where the simple clipping approach failed, (3) successfully avoided the potential conceptual ambiguities that are implied by those ad hoc down-regulation approaches. We expect our approach will make future biogeochemical models easier to improve and more robust.
\end{abstract}

\section{Introduction}

Biogeochemical modeling has been one of the major themes in developing Earth system models (Hurrell et al., 2013), yet developing numerically robust and mathematically consistent biogeochemical (BGC) models has been challenging (Broekhuizen et al., 2008). In biogeochemical modeling, the systems of interest, such as terrestrial ecosystems, are often nutrient limited under a wide range of conditions ( $\mathrm{Vi}$ tousek and Howarth, 1991; Vitousek et al., 2010). Therefore, proper modeling of nutrient limitation is a prerequisite for credible predictions of carbon-climate feedbacks (Bouskill et al., 2014; Thomas et al., 2015). In the Earth system models (ESMs) joining phase 5 of the Coupled Model Intercomparison Project (CMIP5), only CLM-CN (Thornton et al., 2007) considered carbon and nitrogen interactions, although observations indicate nitrogen has significantly limited the terrestrial carbon sink (Arora et al., 2013). Further, many analyses indicate phosphorus is critical for improving carbon-climate feedback predictions (Vitousek et al., 2010; Yang et al., 2014; Wieder et al., 2015), and other nutrients (e.g., sulfur, potassium, molybdenum) may also be important (Schmidt et al., 2013; Moro et al., 2014). Therefore, we expect that as more processes are included in future biogeochemical models, more substrates will limit different biogeochemical processes under different conditions.

To develop numerically accurate biogeochemical models, it is important to develop a robust formulation of the biogeochemical processes, such that modelers can safely add or remove biogeochemical processes without degrading the numerical solution. This capability would allow users to focus only on deriving the governing ordinary differential equations (ODEs) of the biogeochemical processes. If the model uses a standard operator-splitting approach (as is common, 
e.g., Tang et al., 2013), which solves the transport and chemistry separately, then the numerical solver could resolve the numerical details, such as maintaining mass conservation and avoiding nonphysical values, without knowing the details of the ODEs.

Existing terrestrial biogeochemical models often describe substrate limitation as occurring when the total available substrate cannot satisfy the demand from all consuming fluxes over a particular time step. For nitrogen limitation, many BGC models impose substrate limitation when the total potential ecosystem nitrogen demand (i.e., demand in the absence of nitrogen limitation; Thornton et al., 2007; Wang et al., 2010; Thomas et al., 2015) exceeds the total available mineral nitrogen, provided nitrogen from nitrogen fixers is supplied in mineral form over that time step and contributions from organic nitrogen are assumed negligible (the latter of which could be incorrect; see Chapin et al., 1993). However, this conceptual model (which served as the basis for those ad hoc down-regulation approaches) is not mathematically consistent with the ODE that governs nitrogen limitation:

$\frac{\mathrm{dN}_{\text {min }}}{\mathrm{d} t}=\mathrm{N}_{\text {min, sup }}-\mathrm{N}_{\text {min, up }}$,

where $\mathrm{N}_{\min }(\mathrm{gN}), \mathrm{N}_{\min \text {, sup }}\left(\mathrm{gN} \mathrm{s}^{-1}\right)$, and $\mathrm{N}_{\min \text {, up }}\left(\mathrm{gN} \mathrm{s}^{-1}\right)$ represent mineral nitrogen, mineral nitrogen supply (e.g., fixation, deposition), and mineral nitrogen uptake, respectively. Mathematically, Eq. (1) implies that nitrogen limitation occurs only when the numerical approximation to $\mathrm{N}_{\text {min }}(t+\Delta t)$ is negative after accounting for mineral nitrogen supply and demand over the numerical time step $\Delta t$. Therefore, considering that negative mineral nitrogen concentration is unphysical, imposing that nitrogen limitation should be mathematically interpreted as a means to ensure $\mathrm{N}_{\text {min }}(t+\Delta t)=\left(\mathrm{N}_{\text {min, sup }}-\mathrm{N}_{\text {min, up }}\right) \Delta t+\mathrm{N}_{\min }(t) \geq 0$, rather than imposing the constraint $\mathrm{N}_{\min , \text { up }} \Delta t \leq \mathrm{N}_{\min }(t)$ as is often done in current BGC models. Another requirement to ensure correct numerical solutions to the ODEs of the biogeochemical model is to maintain mass conservation for different chemical elements involved in the biogeochemical processes. The mass conservation could be violated if one uses the popular clipping method (e.g., Sandu, 2001) to reset negative solutions or by setting the derivative of the negative tobe variable to zero (i.e., the variable will become negative if the derivative is not constrained), as is done for some explicit ODE solvers. For example, the MATLAB function odenonnegative, which is used in the explicit solver ODE45 to avoid negative solutions, resets the derivative for a negative to-be variable to zero. In either implementation, clipping will artificially introduce new mass into the model and such mass will accumulate and grow throughout the model integration, resulting in incorrect model predictions (Sandu et al., 2001).

In this note, we show that, by ensuring mass conservation and non-negative solutions to the governing equations of a given biogeochemical model, it is possible to obtain a uni- versal solution to the mass limitation for an arbitrary number of substrates. We organize the remainder of this paper as follows: Sect. 2 describes the technical details of our method, Sect. 3 presents an evaluation of the method based on a CENTURY-like organic matter decomposition model (Parton et al., 1988; Appendix A, Tables 1 and 2), and Sect. 4 summarizes our findings. Note that, even though our evaluation of the approach is based on a soil biogeochemical model, the approach is generic and could be applied to any biogeochemical models.

\section{Methods}

Our approach makes use of the reaction-based formulation of a biogeochemical model (e.g., Reichert et al., 2001; Batstone et al., 2002; Fang et al., 2013). Mathematically, for the $j$ th reaction, we have

$$
\sum_{i} v_{i, j}^{-} A_{i, j} \rightarrow \sum_{m} v_{m, j}^{+} B_{m, j}
$$

where $v_{i, j}^{-}$and $v_{m, j}^{+}$are stoichiometric coefficients for the $i$ th reactant $A_{i, j}$ and $m$ th product $B_{m, j}$, respectively. Hereafter we assume the units of all chemical species are consistently defined depending on the specific problem.

By defining reaction rate $r_{j}$ of the $j$ th reaction as the consumption rate of the master species in Eq. (2), for instance, $A_{1, j}$, whose stoichiometric coefficient is one, we calculate the temporal variation of any chemical species due to the $j$ th reaction as

$$
\left(\frac{\mathrm{d} x_{i}}{\mathrm{~d} t}\right)_{j}=v_{i, j} r_{j},
$$

where $v_{i, j}$ is the stoichiometric coefficient for chemical $x_{i}$ in the $j$ th reaction. For reactants, $v_{i, j}$ is negative, and for products $v_{i, j}$ is positive; $v_{i, j}$ is zero when a chemical species is not involved in the reaction.

We describe the generic model structure using the Peterson matrix form (e.g., Russell, 2006) as

$$
\frac{\mathrm{d} \boldsymbol{x}}{\mathrm{d} t}=\mathbf{S} \boldsymbol{r},
$$

where $\mathbf{S}=\left\{v_{i, j}\right\}$ is the matrix of stoichiometric coefficients and $\boldsymbol{x}$ and $\boldsymbol{r}$ are vectors of the state variables and reaction rates, respectively.

We now separate $\mathbf{S}$ into two parts, $\mathbf{S}^{+}$and $\mathbf{S}^{-}$, which, respectively, contain product $\left(v_{i, j}^{+} ;>0\right)$ and reactant $\left(v_{i, j}^{-} ; \geq 0\right)$ stoichiometric coefficients, such that

$$
\frac{\mathrm{d} \boldsymbol{x}}{\mathrm{d} t}=\left(\mathbf{S}^{+}-\mathbf{S}^{-}\right) \boldsymbol{r} .
$$

In finding the numerical solution to Eq. (5) over a certain time step, if some reaction rates are too high, certain state 
variables will become negative unless those reaction rates are reduced.

Several approaches (other than clipping) have been proposed to ensure non-negative and mass conservative solutions to equations such as Eq. (5). For instance, Sandu (2001) proposed two projection-based approaches to post-correct the negative solution using the null space of $\mathbf{S}^{T}$ (here $T$ denotes transpose). Although his approaches overcome the barriers that non-negativity restricts either the order of the method to 1 or the step size to impractically small values (Bolley and Crouzeix, 1978), they require matrix inversion, which may become computationally intensive as the problem size increases or impractical because not every model formulation allows negative state variables in the intermediate step when a high-order scheme is employed. Broekhuizen et al. (2008) ensured the solution non-negativity of Eq. (5) by applying a single flux limiter (i.e., a scalar modifier that reduces the reaction rate) to all reaction rates in the governing equations (i.e., the mBBKS scheme). However, as we will show below, the mBBKS approach will fail for models such as CENTURY-like organic matter decomposition models (Parton et al., 1988; Appendix A, Table 1) when multiple substrates are limiting under different conditions. We also note that the occurrence of a negative solution is not unique to the CENTURY-like model that calculates the reaction rates using the linear kinetics. Michaelis-Menten kinetics-based soil biogeochemical models (e.g., Gerber et al., 2010; Bouskill et al., 2012) would similarly suffer from the negative-solution problem when many substrates could limit the reaction rates, and such a problem cannot be easily solved by simply resorting to adaptive time-stepping algorithms; therefore, the solution strategy proposed below resolves a common issue for any biogeochemical models.

To propose a simple solution to ensure non-negative numerical solutions to Eq. (5), we restrict our ODE integrator to the first order and apply a vector of flux limiters that are dependent on the reactant stoichiometry $\mathbf{S}^{-}$, which controls the total substrate demand. Forcing the flux limiter solution to depend linearly on $\mathbf{S}^{-}$maintains the stoichiometric relationship for all reactions and thus mass balance over the time step. Specifically, we calculate and apply the flux limiter for each reaction according to the following Fortran 90 code:

!M1 is number of state variables.

!N1 is number of reactions.

$! x t$ is vector of state variables at current time step.

!xtnew is vector of temporary state variables for next time step.

$! q$ is vector of flux limiters for all reactions.

!d $t$ is time step size.

$\ln$ eg $=$.false. !Initialize negative state variable indicator to zero

do $m=1, M 1$ ! Loop over all state variables

xtnew $(m)=x t(m)$

$F p=0.0$ !Initialize production flux accumulator to zero

$F m=0.0$ !Initialize consumption flux accumulator to zero

do $n=1, N 1$ ! Loop over all reactions xtnew $(m)=x \operatorname{tnew}(m)+(\operatorname{sp}(m, n)-\operatorname{sm}(m, n))$ $* r(n) * \mathrm{~d} t$

$F p=F p+s p(m, n) * r(n)$

$F m=F m+s m(m, n) * r(n)$

enddo

if $(x$ tnew $(m)<0)$ then !The state variable tends to be negative

!Calculate the limiting factor

$p(m)=(x t(m)+F p * \mathrm{~d} t) /(\mathrm{d} t * F m)$

lneg $=$.true.

endif

enddo

!Now compute and apply the flux limiter

!when there is any negative state variable if (lneg) then

do $n=1, N 1$

$! \min p$ finds the minimum of $\mathrm{p}$,

!where the corresponding entry in $s m$ is $>0$.

$q(n)=\min p(p(1: M 1), \operatorname{sm}(1: M 1, n))$

$r(n)=r(n) * q(n)$

endif

enddo

The function minp is defined in Appendix B. In rare situations, one has to apply the above flux-limiting procedure several times to ensure solution non-negativity, but the computation is much quicker and simpler than the matrix inversion required in Sandu's projection methods (2001), and can be paralleled easily. In addition, for a single biogeochemical reaction, one can verify that our approach is equivalent to Liebig's law of the minimum as applied to a generic biogeochemical reaction, which can be re-stated for a highfrequency $\mathrm{BGC}$ model to imply that the mean reaction rate during a numerical time step is controlled by the most limiting substrate. It is also noted that our approach avoids the explicit formulation of the law of the minimum in calculating the reaction rates, as is often done in many existing biogeochemical models (e.g., CLM-CNP; Yang et al., 2014), which when combined with their ad hoc down-regulation method leads to double counting of substrate limitation. We further note that traditional ODE solvers only require the temporal derivatives of the state variables from the biogeochemical model. Application of our approach in an ODE solver, however, requires the biogeochemical model to return the reaction rates as well as the positive and negative parts of the stoichiometry matrix $\mathbf{S}$. 
Table 1. A list of biogeochemical reactions as represented in the CENTURY-like organic matter decomposition model (Appendix A). The decomposition is calculated as in Parton et al. (1988). Here we use CN to represent carbon to nitrogen ratio, and CP to represent carbon to phosphorus ratio. The subscript "min" designates mineral pool for a nutrient, such as nitrogen (N) and phosphorus (P).

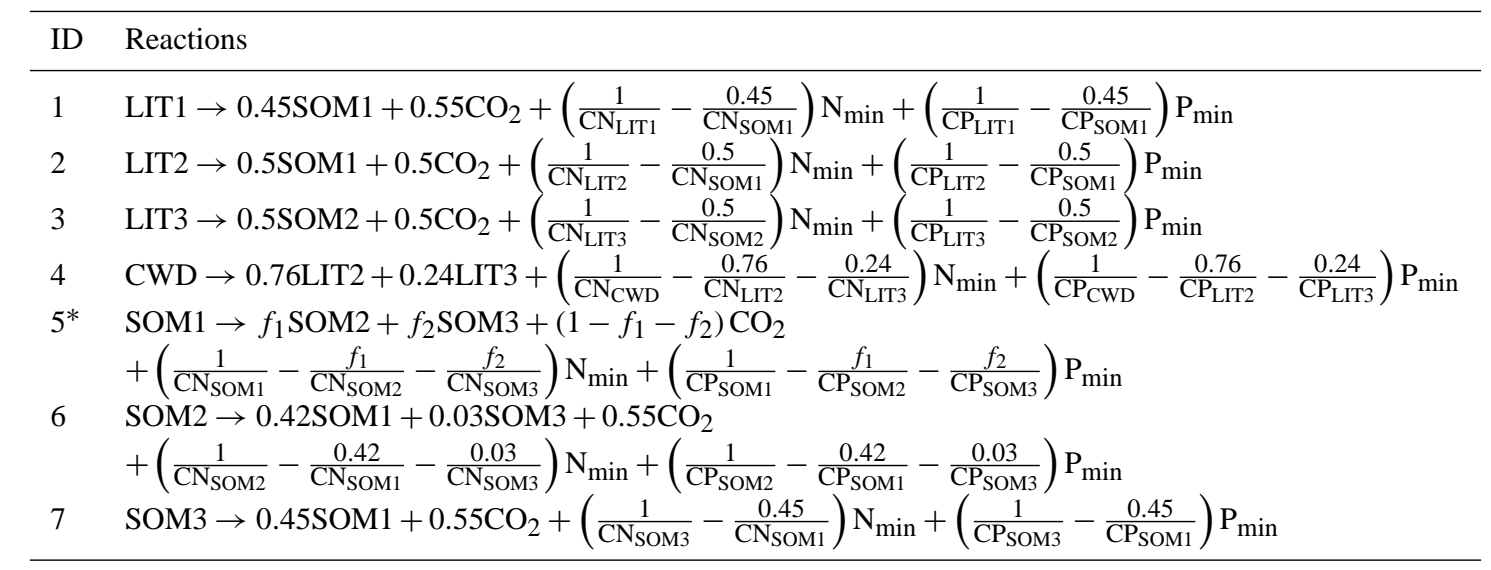

* In this study, we set $f_{1}=0.6235$ and $f_{2}=0.0025$.

Table 2. Parameter values used in this study. These values are based on syntheses from Parton et al. (1988), Yang et al. (2014), and Zhu et al. (2016).

\begin{tabular}{|c|c|}
\hline Parameters & Values \\
\hline$\left(\mathrm{CN}_{\mathrm{LIT} 1}, \mathrm{CP}_{\mathrm{LIT} 1}\right)$ & $(90,1600)$ \\
\hline$\left(\mathrm{CN}_{\text {LIT2 }}, \mathrm{CP}_{\text {LIT2 }}\right)$ & $(90,2000)$ \\
\hline$\left(\mathrm{CN}_{\text {LIT3 }}, \mathrm{CP}_{\text {LIT3 }}\right)$ & $(90,2500)$ \\
\hline$\left(\mathrm{CN}_{\mathrm{CWD}}, \mathrm{CP}_{\mathrm{CWD}}\right)$ & $(90,4500)$ \\
\hline$\left(\mathrm{CN}_{\mathrm{SOM} 1}, \mathrm{CP}_{\mathrm{SOM} 1}\right)$ & $(13,110)$ \\
\hline$\left(\mathrm{CN}_{\mathrm{SOM} 2}, \mathrm{CP}_{\mathrm{SOM} 2}\right)$ & $(16,320)$ \\
\hline$\left(\mathrm{CN}_{\mathrm{SOM} 3}, \mathrm{CP}_{\mathrm{SOM} 3}\right)$ & $(7.9,114)$ \\
\hline
\end{tabular}

In our evaluation, we compared the performance of our new approach to the mBBKS approach (Broekhuizen et al., 2008) and two ad hoc down-regulation formulations derived based on the nitrogen limitation scheme in CLM4.5 (CLM-1 and CLM-2). During a particular numerical time step, CLM1 assumes complete independence between nutrient mobilizers and immobilizers, while CLM-2 assumes complete coupling between nutrient mobilizers and immobilizers (see details in Appendix C). We analyzed scenarios where the organic matter decomposition is (1) not nutrient limited (Case 1) and (2) nitrogen and phosphorus limited (Case 2 and Case 3 ); the latter situations are where a direct solution (without flux limitation) to Eq. (5) may produce negative values, and clipping will be triggered in methods like ODE45. We evaluated the difference between simulations for predicted mineral nitrogen $\mathrm{N}_{\text {min }}$, mineral phosphorus $P_{\text {min }}$, total litter carbon, and total soil organic carbon. We note that all litter-decomposing reactions in the CENTURY-like model immobilize nitrogen and phosphorus; therefore, when SOM pools (SOM1, SOM2, and SOM3) are zero, a non-zero pool size must be assigned to both soil mineral nitrogen and min- eral phosphorus to initialize litter decomposition (such as for Case 2 and Case 3; Table 3). We describe the initial conditions for our model runs in Table 3: Case 1 represents nutrient non-limiting decomposition, Case 2 represents nutrientlimited decomposition with zero initial SOM pools, and Case 3 represents nutrient-limited decomposition with non-zero initial SOM pools. We also conducted Case 4 to reveal that the conceptual ambiguity in those ad hoc down-regulation approaches will result in model uncertainties that could be avoided in our new approach. Case 4 differs from Case 3 with the addition of a first-order loss term for both mineral nitrogen and mineral phosphorus and a continuous litter input for the first 1500 days of the 3000-day integration (Table 3). These mineral nutrient loss terms are used to mimic nutrient demand from other processes as would occur in a BGC model in ESMs. Because there are no analytical solutions to the CENTURY-like model, Case 1 also serves as a benchmark for our implementation of different numerical solution strategies with respect to ODE45, which has been very well tested by the MATLAB developers for problems that have no non-negativity constraint on their solutions. We coded all our methods as MATLAB scripts and all ODE integrations are carried out using an adaptive time-stepping strategy (Appendix D) with a relative error $10^{-4}$.

\section{Results and discussions}

\subsection{Method evaluation}

In simulations for the decomposition of nutrient-sufficient organic matter (i.e., no nutrient limitation; Fig. 1), we found that our new approach (Fortran 90 code, Eq. 6), mBBKS, CLM-1, and CLM-2 predicted almost identical time series for the various pools when compared to that from ODE45, in- 

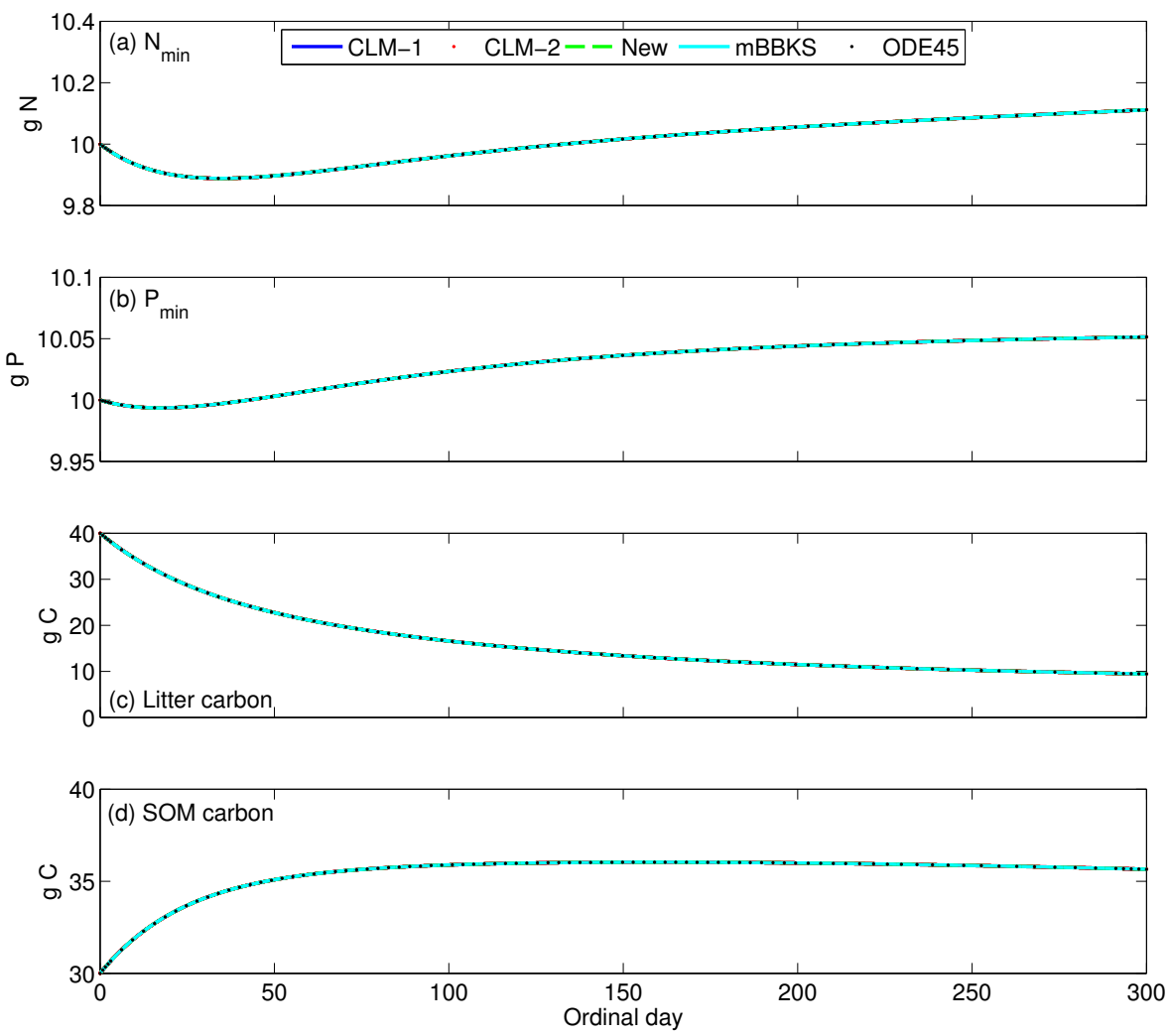

Figure 1. Simulated decomposition dynamics for Case 1 in Table 3. In all panels, all results overlap each other.

dicating that the four approaches are implemented correctly as benchmarked with ODE45.

However, for Case 2 (Fig. 2, Table 3), where both nitrogen and phosphorus are insufficient to support decomposition (because it has even fewer mineral nutrients available than the nutrient-limited Case 3), mBBKS failed to predict any organic matter decomposition after the mineral nutrients are consumed in the first few time steps and predicted that all decomposition pathways were phosphorus limited thereafter (cyan line in Fig. 2b). In contrast, the two ad hoc downregulation approaches, CLM-1 and CLM-2, and our new approach all predicted visually identical time series of the different pools and correctly indicated that the decomposition of SOM pools (SOM1, SOM2, and SOM3 as derived from litter decomposition using the non-zero initial pools of mineral nutrients) released small amounts of mineral nutrients to support further litter organic matter decomposition (as can be inferred from Table 2, which shows that the decomposition of litter pools are all nutrient limited by stoichiometry). This response was missed by mBBKS, because it applied a single flux limiter to all decomposition pathways, preventing the release of nutrients from mineralizing pathways to support further decomposition. Besides mBBKS, ODE45 also failed to predict meaningful decomposition dynamics because, by clipping the derivatives of the negative to-be state variables to zero, it introduced artificial mass into some state vari- ables during the integration. Specifically, ODE45 predicted the final total nitrogen and total phosphorus (including both mineral and organic pools) as $0.8066 \mathrm{gN}$ and $0.0511 \mathrm{gP}$, as compared to the correct values of $0.4445 \mathrm{gN}$ and $0.0175 \mathrm{gP}$, whereas CLM-1, CLM-2, and our new approach all conserved carbon, nitrogen, and phosphorus mass within the machine round-off error.

For Case 3 (Fig. 3, Table 3), where non-zero SOM pools were introduced to release nitrogen and phosphorus to support litter decomposition, mBBKS again predicted no visible decomposition because of its use of a single flux limiter to all fluxes (based on the nutrient-limited litter decomposition), even though the SOM decomposition should not be nutrient limited. ODE45 also failed for Case 3 , and predicted very different time series for the various pools as compared to CLM1, CLM-2, and our new approach. By day 300, ODE45 predicted the total nitrogen and total phosphorus (including both mineral and organic pools) as $3.2164 \mathrm{gN}$ and $0.2338 \mathrm{gP}$ as compared to their correct values of $3.1046 \mathrm{gN}$ and $0.2273 \mathrm{gP}$, respectively.

\subsection{The conceptual ambiguity of implementing nutrient limitation}

Although we found very small differences between our new method, CLM-1, and CLM-2 in predicted decomposition 

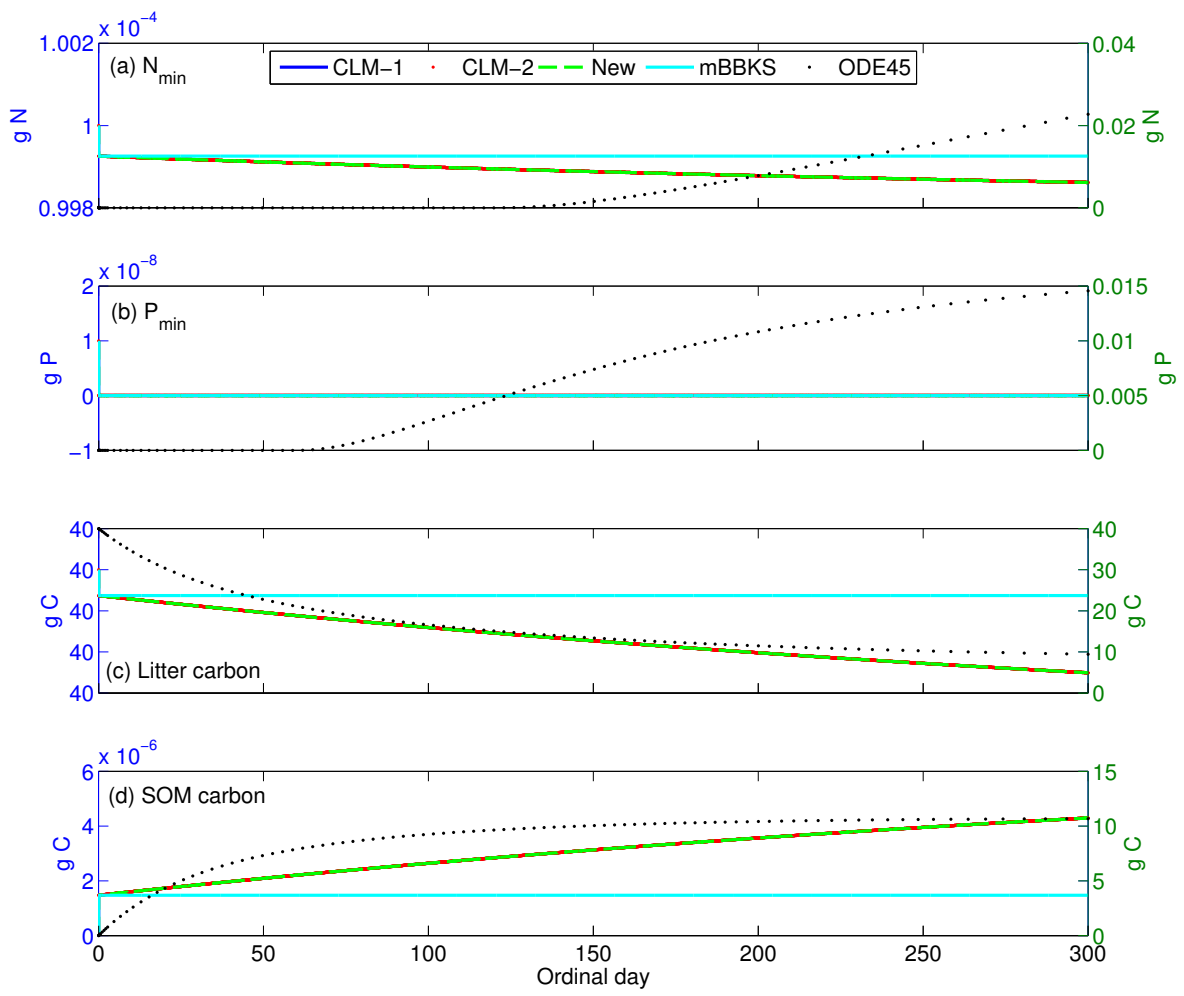

Figure 2. Simulated decomposition dynamics for Case 2 in Table 3. Note that the ODE45 scheme (shown on the right-hand $y$ axes) predicted wrong results that are of much large magnitude than the other methods.

Table 3. Initial conditions and integration length (\#days) for the analyzed model simulations.

\begin{tabular}{|c|c|c|c|c|}
\hline Variables & Case 1 & Case 2 & Case 3 & Case $4^{*}$ \\
\hline LIT1 & $10 \mathrm{gC}$ & $10 \mathrm{gC}$ & $10 \mathrm{gC}$ & $10 \mathrm{gC}$ \\
\hline LIT2 & $10 \mathrm{gC}$ & $10 \mathrm{gC}$ & $10 \mathrm{gC}$ & $10 \mathrm{gC}$ \\
\hline LIT3 & $10 \mathrm{gC}$ & $10 \mathrm{gC}$ & $10 \mathrm{gC}$ & $10 \mathrm{gC}$ \\
\hline CWD & $10 \mathrm{gC}$ & $10 \mathrm{gC}$ & $10 \mathrm{gC}$ & $10 \mathrm{gC}$ \\
\hline SOM1 & $10 \mathrm{gC}$ & $0 \mathrm{gC}$ & $10 \mathrm{gC}$ & $10 \mathrm{gC}$ \\
\hline SOM2 & $10 \mathrm{gC}$ & $0 \mathrm{gC}$ & $10 \mathrm{gC}$ & $10 \mathrm{gC}$ \\
\hline SOM3 & $10 \mathrm{gC}$ & $0 \mathrm{gC}$ & $10 \mathrm{gC}$ & $10 \mathrm{gC}$ \\
\hline $\mathrm{N}_{\min }$ & $10 \mathrm{~g} \mathrm{~N}$ & $10^{-4} \mathrm{~g} \mathrm{~N}$ & $10^{-4} \mathrm{~g} \mathrm{~N}$ & $10^{-3} \mathrm{gN}$ \\
\hline $\mathrm{P}_{\min }$ & $10 \mathrm{~g} \mathrm{P}$ & $10^{-8} \mathrm{gP}$ & $10^{-8} \mathrm{gP}$ & $10^{-7} \mathrm{gP}$ \\
\hline$q_{\mathrm{Nmin}}$ & $0 s^{-1}$ & $0 s^{-1}$ & $0 s^{-1}$ & $10^{-6} \mathrm{~s}^{-1}$ \\
\hline$q_{\text {Pmin }}$ & $0 \mathrm{~s}^{-1}$ & $0 s^{-1}$ & $0 s^{-1}$ & $10^{-6} \mathrm{~s}^{-1}$ \\
\hline \#days & 300 days & 300 days & 300 days & 3000 days \\
\hline
\end{tabular}

* For Case 4, there were rates of $0.04,0.04$, and $0.02 \mathrm{~g} \mathrm{C}$ day $^{-1}$ input for LIT1, LIT2, and LIT3 in the first 1500 days.

dynamics for the three simple cases analyzed (Figs. 1, 2, and 3), we acknowledge that large differences should be expected when applying our new method and the two ad hoc down-regulation approaches CLM-1 and CLM-2 for modeling ecosystem dynamics because they define nutrient limitation differently (Fortran 90 code, Eq. 6, and Appendix C;
Figs. 4 and 5). As one would infer from Eq. (1), mathematically, nutrient limitation occurs only when the state variable that represents a certain nutrient becomes negative if the reaction rates are not limited during a given numerical integration time step. However, (as we implemented in the Fortran 90 code, Eq. 6), this situation is equivalent to assuming that a released mineral nutrient from the mobilizers will be instantaneously available to all immobilizers that demand this nutrient. Although the existing mineral nutrient pool and the newly released mineral nutrients will be tapped proportionally by the immobilizers, this assumption may still be too strong if a given grid cell covers a too large spatial domain to support this assumption of homogeneity (Manzoni et al., 2008). CLM-1 and CLM-2 represent the two extremes of this coupling between mineral nutrient mobilizers (which release nutrients) and mineral nutrient immobilizers (which take up nutrients) in that CLM-1 assumes the mobilizers and immobilizers are completely independent during the calculation of mineral nutrient uptake, whereas CLM-2 assumes the nutrients released by mobilizers are first assimilated by immobilizers, and if there is additional demand, the remainder comes from the mineral nutrient pool (thus CLM-2 adopts an even stronger mobilizer and immobilizer coupling than our new approach). Indeed, the difference between CLM-1 and CLM2 is already discernible for Case 3 (Fig. 4), and when the decomposition model is coupled with other nutrient consumers 

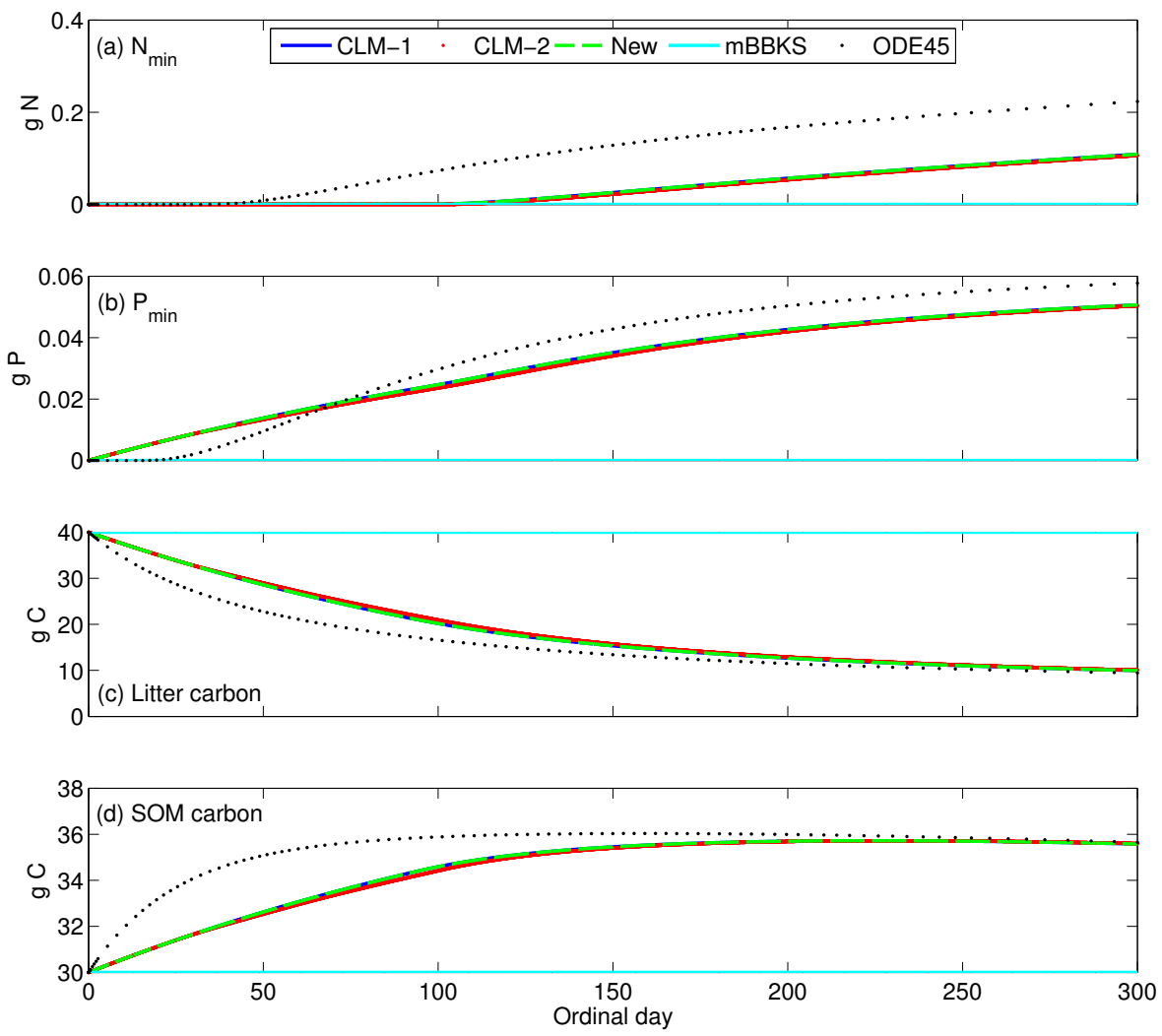

Figure 3. Simulated decomposition dynamics for Case 3 in Table 3. In all panels, the result from CLM-1 overlaps with that from our new method.

in an ecosystem model, one would potentially find very different predictions of carbon dynamics (see Case 4 in Fig. 5 as a model with slightly more complicated dynamics than Case $3)$. With slight modification, our new approach will allow a consistent representation of the coupling between mobilizers and immobilizers, including both the CLM-1 and CLM-2 assumptions regarding nutrient competition. This approach will provide a new tool to analyze prediction uncertainty from the ambiguity of defining the coupling strength between nutrient mobilizers and immobilizers.

Another advantage of our new approach, compared to the ad hoc down-regulation approaches (e.g., CLM-1 and CLM2 discussed above), is that it can handle limitation from an arbitrary number of substrates, as long as the matrix of stoichiometric coefficients is formulated. In principle, any biogeochemical reaction can be formulated into reaction form (e.g., Fang et al., 2013); thus, our approach will avoid the ordering problem often encountered in those ad hoc approaches. In this context, the "ordering problem" refers to the situation that different answers are calculated depending on the order of nutrient limitation (e.g., resolving nitrogen limitation first, and then phosphorus limitation). For example, following the nutrient limitation definition in CLM-1, when nitrogen and phosphorus limitation are treated sequentially, the predicted decomposition dynamics differ signifi- cantly from when the opposite order is applied (CLM-1NP vs. CLM-1PN in Fig. 5). The implementation where nitrogen limitation occurs before phosphorus limitation (CLM-1NP, cyan circles in Fig. 5) predicted stronger litter decomposition than when phosphorus limitation is applied before nitrogen limitation (CLM-1PN, black dots that overlap with blue line in Fig. 5c). Analogously, in the current CLM4.5 soil biogeochemical formulation (Oleson et al., 2013), organic matter decomposition and methane oxidation are often limited by oxygen (Riley et al., 2011), and nitrogen limitation is imposed after accounting for oxygen limitation, which would potentially result in different predictions were nitrogen limitation imposed before oxygen limitation. This ordering issue could become more severe if more nutrients (e.g., phosphorus, sulfur) are to be introduced in future biogeochemical formulations, and our approach relieves numerical inaccuracies associated with this ordering ambiguity.

\section{Conclusions}

In this study, we proposed a generic law of the minimumbased flux limiter to handle substrate limitation in biogeochemical models. Evaluations indicate that our method could produce results as accurate as those produced by ad hoc 

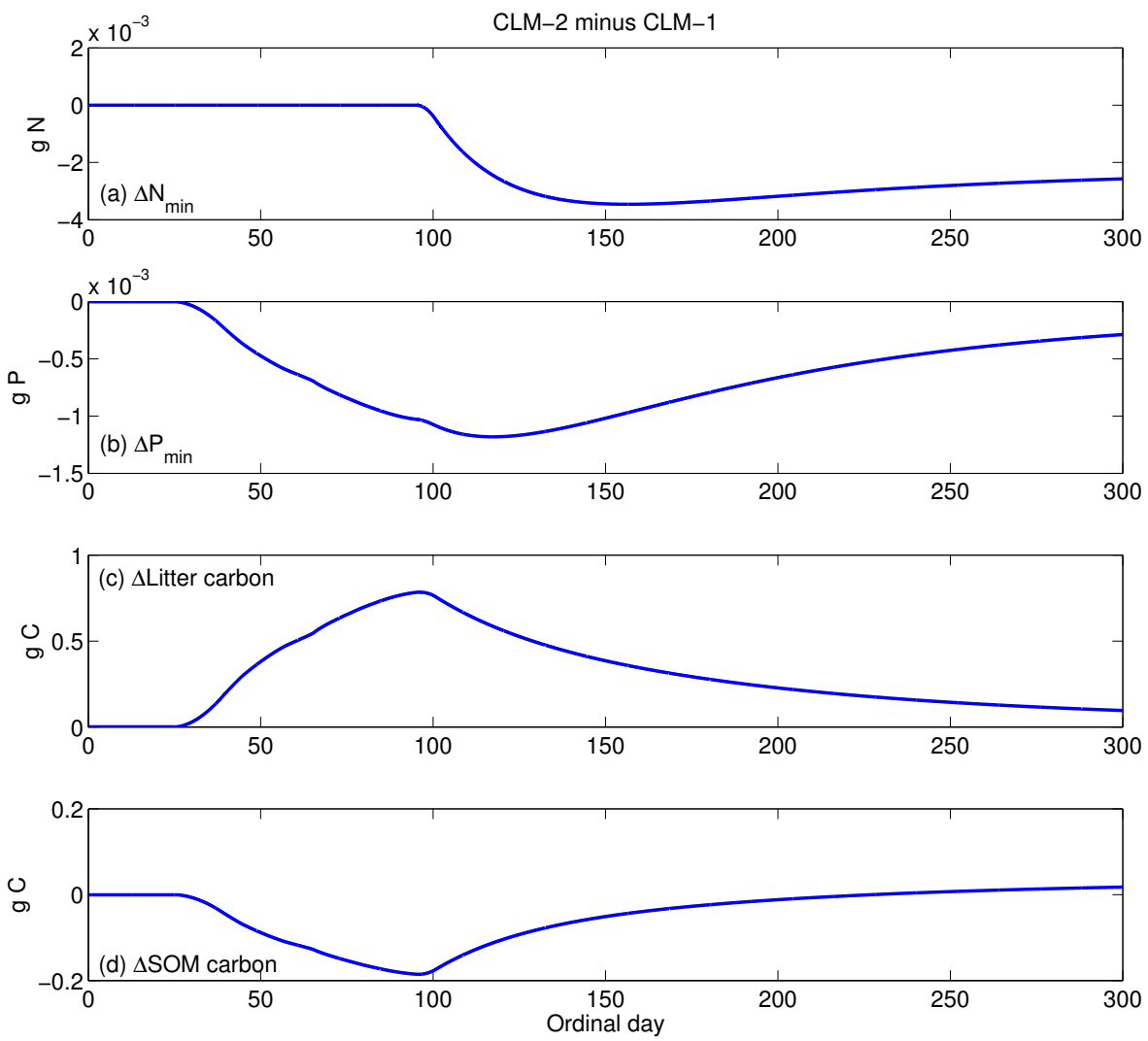

Figure 4. Differences between simulated decomposition dynamics by CLM-1 and CLM-2 for Case 3.

down-regulation approaches implemented in existing biogeochemical models for simple decomposition dynamics that only include decomposers. Additionally, our new approach provides a way to resolve some conceptual ambiguities implied in those ad hoc down-regulation approaches. We expect our new approach to help the community to develop more robust and easier to maintain biogeochemical codes to better predict carbon-climate feedbacks. 

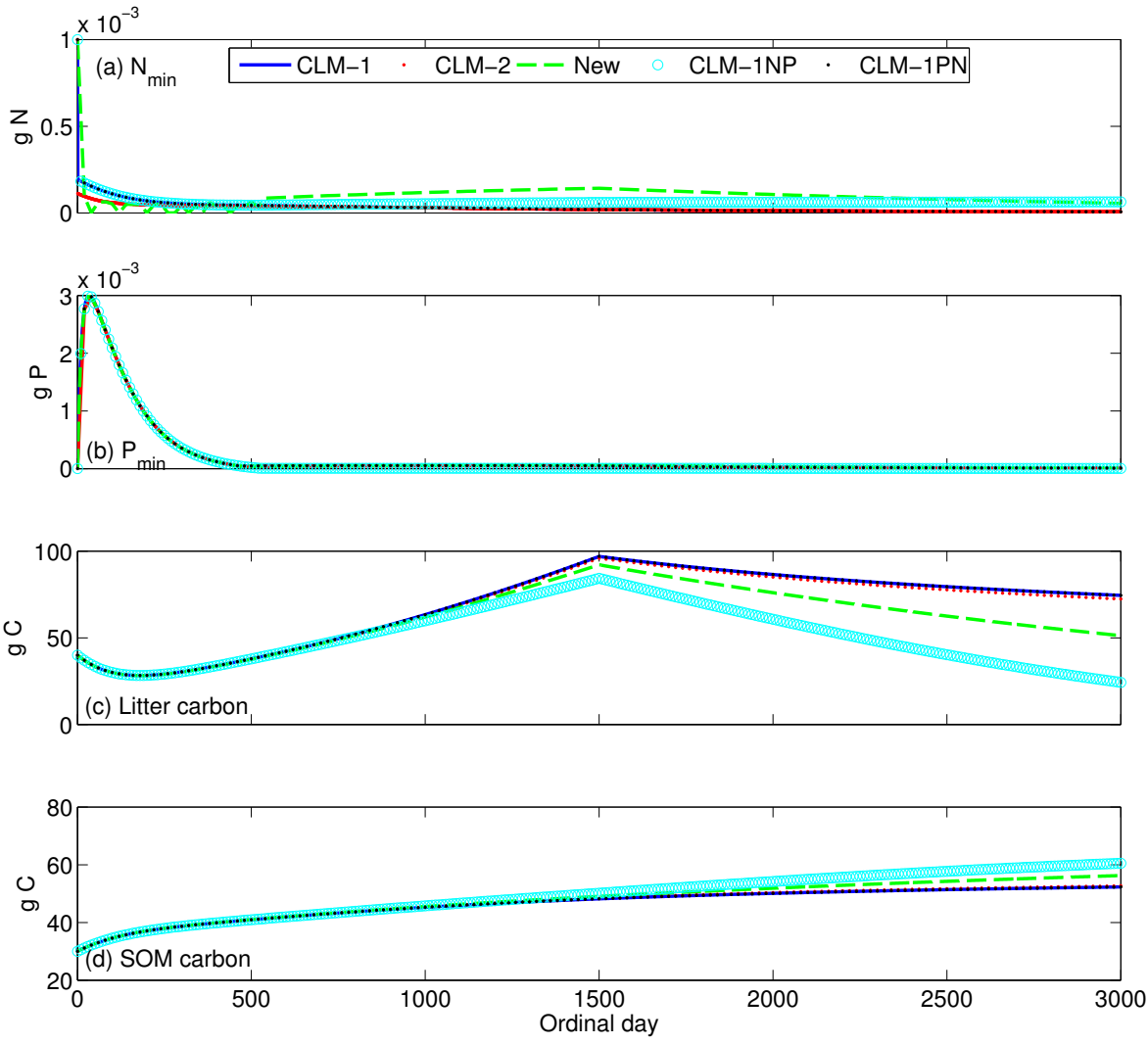

Figure 5. Simulated decomposition dynamics for Case 4 in Table 3. CLM-1NP performs nitrogen down-regulation before phosphorus down-regulation, whereas CLM-1PN reverses the order. Similarly to CLM-1 (Appendix C), both CLM-1NP and CLM-1PN assume the nutrient mobilizer and immobilizer are independent within a numerical time step. In all panels, CLM-1PN predictions overlap with CLM-1 predictions. 


\section{Appendix A: Governing equations for the CENTURY-like decomposition model}

The soil biogeochemical model used in this study adopts the form from the CENTURY-model, which uses a turnoverpool-based formulation of soil organic matter decomposition (Parton et al., 1988). The model includes three pools of litter, one pool of coarse wood debris (CWD) and three pools of SOM. The model calculates the non-nutrient-limited decomposition of a given organic matter pool $X$ using the first-order kinetics, $r_{X}=-k_{X} X$, where $k_{X}\left(\mathrm{yr}^{-1}\right)$ is the decay parameter (and is equal to the reciprocal of the turnover time). In most applications $k_{X}$ is a function of temperature and moisture, however, it is taken as constant in this study. Following CLM4.5 BGC (Oleson et al., 2013), the turnover times are $0.066,0.25$, and 0.25 years, respectively, for the three litter pools LIT1, LIT2 and LIT3. For the three SOM pools, the turnover times are $0.17,6.1$, and 270 years, respectively, for SOM1, SOM2 and SOM3. CWD has a turnover time of 4.1 years. The decomposed organic matter released from linear decay is redistributed through the seven organic matter pools according to the reaction stoichiometry in Table 1. Mathematically, the CENTURY-like decomposition model is summarized with the following governing equations:

$$
\begin{aligned}
& \frac{\mathrm{dLIT} 1}{\mathrm{~d} t}=-r_{\mathrm{LIT} 1}+I_{\mathrm{LIT} 1}, \\
& \frac{\mathrm{dLIT} 2}{\mathrm{~d} t}=-r_{\mathrm{LIT} 2}+0.76 r_{\mathrm{cwd}}+I_{\mathrm{LIT} 2}, \\
& \frac{\mathrm{dLIT3}}{\mathrm{d} t}=-r_{\mathrm{LIT3}}+0.24 r_{\mathrm{cwd}}+I_{\mathrm{LIT} 3}, \\
& \frac{\mathrm{dCWD}}{\mathrm{d} t}=-r_{\mathrm{CWD}}+I_{\mathrm{CWD}} \text {, } \\
& \frac{\mathrm{dSOM} 1}{\mathrm{~d} t}=-r_{\mathrm{SOM} 1}+0.45 r_{\mathrm{LIT} 1}+0.5 r_{\mathrm{LIT} 2} \\
& +0.42 r_{\mathrm{SOM} 2}+0.45 r_{\mathrm{SOM} 3}+I_{\mathrm{SOM} 1} \text {, } \\
& \frac{\mathrm{dSOM} 2}{\mathrm{~d} t}=-r_{\mathrm{SOM} 2}+0.5 r_{\mathrm{LIT} 3}+f_{1} r_{\mathrm{SOM} 1}+I_{\mathrm{SOM} 2}, \\
& \frac{\mathrm{dSOM} 3}{\mathrm{~d} t}=-r_{\mathrm{SOM} 3}+0.03 r_{\mathrm{SOM} 2}+f_{2} r_{\mathrm{SOM} 1}+I_{\mathrm{SOM} 3} \text {, } \\
& \frac{\mathrm{dN}_{\min }}{\mathrm{d} t}=\left(\frac{1}{\mathrm{CN}_{\mathrm{LIT} 1}}-\frac{0.45}{\mathrm{CN}_{\mathrm{SOM} 1}}\right) r_{\mathrm{LIT} 1} \\
& +\left(\frac{1}{\mathrm{CN}_{\mathrm{LIT} 2}}-\frac{0.5}{\mathrm{CN}_{\mathrm{SOM} 1}}\right) r_{\mathrm{LIT} 2} \\
& +\left(\frac{1}{\mathrm{CN}_{\mathrm{LIT} 3}}-\frac{0.5}{\mathrm{CN}_{\mathrm{SOM} 2}}\right) r_{\mathrm{LIT} 3} \\
& +\left(\frac{1}{\mathrm{CN}_{\mathrm{CWD}}}-\frac{0.76}{\mathrm{CN}_{\mathrm{LIT} 2}}-\frac{0.24}{\mathrm{CN}_{\mathrm{LIT} 3}}\right) r_{\mathrm{CWD}} \\
& +\left(\frac{1}{\mathrm{CN}_{\mathrm{SOM} 1}}-\frac{f_{1}}{\mathrm{CN}_{\mathrm{SOM} 2}}-\frac{f_{2}}{\mathrm{CN}_{\mathrm{SOM} 3}}\right) r_{\mathrm{SOM} 1} \\
& +\left(\frac{1}{\mathrm{CN}_{\mathrm{SOM} 2}}-\frac{0.42}{\mathrm{CN}_{\mathrm{SOM} 2}}-\frac{0.03}{\mathrm{CN}_{\mathrm{SOM} 3}}\right) r_{\mathrm{SOM} 2}
\end{aligned}
$$

$$
\begin{aligned}
& +\left(\frac{1}{\mathrm{CN}_{\mathrm{SOM} 3}}-\frac{0.45}{\mathrm{CN}_{\mathrm{SOM} 1}}\right) r_{\mathrm{SOM} 3}-q_{\mathrm{N}} \min \mathrm{N}_{\mathrm{min}}, \\
& \frac{\mathrm{dP}_{\mathrm{min}}}{\mathrm{d} t}=\left(\frac{1}{\mathrm{CP}}-\frac{0.45}{\mathrm{CIT} 1}\right) r_{\mathrm{LIT} 1} \\
& +\left(\frac{1}{\mathrm{CP}_{\mathrm{LIT} 2}}-\frac{0.5}{\mathrm{CP}}\right) r_{\mathrm{LITM} 2} \\
& +\left(\frac{1}{\mathrm{CP}_{\mathrm{LIT} 3}}-\frac{0.5}{\mathrm{CP}_{\mathrm{SOM} 2}}\right) r_{\mathrm{LIT} 3} \\
& +\left(\frac{1}{\mathrm{CP}_{\mathrm{CWD}}}-\frac{0.76}{\mathrm{CP}_{\mathrm{LIT} 2}}-\frac{0.24}{\mathrm{CP}_{\mathrm{LIT} 3}}\right) r_{\mathrm{CWD}} \\
& +\left(\frac{1}{\mathrm{CP}_{\mathrm{SOM} 1}}-\frac{f_{1}}{\mathrm{CP}_{\mathrm{SOM} 2}}-\frac{f_{2}}{\mathrm{CP}_{\mathrm{SOM} 3}}\right) r_{\mathrm{SOM} 1} \\
& +\left(\frac{1}{\mathrm{CP}_{\mathrm{SOM} 2}}-\frac{0.42}{\mathrm{CP}_{\mathrm{SOM} 2}}-\frac{0.03}{\mathrm{CP}_{\mathrm{SOM} 3}}\right) r_{\mathrm{SOM} 2} \\
& +\left(\frac{1}{\mathrm{CP}_{\mathrm{SOM} 3}}-\frac{0.45}{\mathrm{CP}_{\mathrm{SOM} 1}}\right) r_{\mathrm{SOM} 3}-q_{\mathrm{P}} \min \mathrm{P}_{\min }
\end{aligned}
$$

where $q_{\mathrm{N} \text { min }}\left(\mathrm{s}^{-1}\right)$ and $q_{\mathrm{P} \min }\left(\mathrm{s}^{-1}\right)$ are, respectively, the loss rates for mineral nitrogen and mineral phosphorus, and $I_{(X)}$ $\left(\mathrm{gC} \mathrm{s}^{-1}\right)$ designates the input rate for organic matter $X$. All other symbols are explained in Table 1 .

\section{Appendix B: Pseudo-code for minp}

For two vectors $x$ and $y$ of size $n, p=\min p(x, y)$ (assuming $p \leq 1)$ is calculated as

$$
\begin{aligned}
& p=1 \\
& \text { do } i=1, n \\
& \text { if }(y(i)>0) \text { then } \\
& \quad p=\min (x(i), p) \\
& \text { endif } \\
& \text { enddo. }
\end{aligned}
$$

\section{Appendix C: Two ad hoc down-regulation formulations of nutrient limitation}

The first ad hoc down-regulation approach (CLM-1) follows the implementation of nitrogen down-regulation in CLM4.5 (Oleson et al., 2013), where all nitrogen immobilization fluxes $\mathrm{N}_{\text {immob }}$ within the time step $\Delta t$ are summed and compared to available nitrogen $\left(\mathrm{N}_{\min }\right)$. The flux limiter from mineral nitrogen is

$\gamma_{\mathrm{N}}=\min \left(\frac{\mathrm{N}_{\min }}{\mathrm{N}_{\text {immob }} \Delta t}, 1\right)$.

Similarly, for mineral phosphorus,

$\gamma_{\mathrm{P}}=\min \left(\frac{\mathrm{P}_{\min }}{\mathrm{P}_{\mathrm{immob}} \Delta t}, 1\right)$.

Then for reactions (Table 1) that are only nitrogen limited, we multiply their reaction rates by $\gamma_{\mathrm{N}}$; for reactions that are 
only phosphorus limited, we multiply their reaction rates by $\gamma_{\mathrm{P}}$; and for reactions that are both nitrogen and phosphorus limited, we multiply their reaction rates by $\min \left(\gamma_{\mathrm{N}}, \gamma_{\mathrm{P}}\right)$.

The second ad hoc down-regulation approach (CLM-2) is similar to the first one, except that it first subtracts the mobilizing fluxes from the immobilizing fluxes, such that

$$
\begin{aligned}
& \gamma_{\mathrm{N}}=\min \left(\max \left(\frac{\mathrm{N}_{\text {min }}}{\left(\mathrm{N}_{\mathrm{immob}}-\mathrm{N}_{\mathrm{mob}}\right) \Delta t}, 0\right), 1\right), \\
& \gamma_{P}=\min \left(\max \left(\frac{P_{\text {min }}}{\left(P_{\mathrm{immob}}-P_{\mathrm{mob}}\right) \Delta t}, 0\right), 1\right) .
\end{aligned}
$$

Therefore, it can be inferred that (under similar conditions) nutrient limitation would (theoretically) occur more frequently in the first (CLM-1) than in the second (CLM-2) ad hoc down-regulation approach.

One can further define

$\gamma_{N}=\min \left(\max \left(\frac{\mathrm{N}_{\text {min }}+\mathrm{N}_{\mathrm{mob}} \Delta t}{\mathrm{~N}_{\mathrm{immob}} \Delta t}, 0\right), 1\right)$,

$\gamma_{P}=\min \left(\max \left(\frac{P_{\mathrm{min}}+P_{\mathrm{mob}} \Delta t}{P_{\mathrm{immob}} \Delta t}, 0\right), 1\right)$,

which can be verified to be identical to that calculated in the pseudo-code (Eq. 6) in the main text when only one nutrient is limiting. Because this last definition does not change the conclusion of our study, we only analyzed the first two ad hoc down-regulation approaches (CLM-1 and CLM-2) in our comparison.

\section{Appendix D: MATLAB pseudo-code for the adaptive time-stepping integration}

For a certain time step

\%hscal is the time step scaling factor.

$\% x_{\text {old }}$ is the state variable at current time step.

$\% t$ is current time, and $\Delta t$ is time step.

$x_{\text {new }}=$ ode $\left(x_{\text {old }}, \Delta t, t\right)$

$x_{\text {new }}^{*}=$ ode $\left(x_{\text {old }}, \Delta t / 2, t\right)$

$x_{\text {new }}^{*}=\operatorname{ode}\left(x_{\text {new }}^{*}, \Delta t / 2, t+\Delta t / 2\right)$

$\%$ Find the maximum relative error across all state variables.

$$
\begin{aligned}
& \text { rerr }=\max _{i}\left\{\frac{\left|x_{\text {new }}^{*}(i)-x_{\text {new }}(i)\right|}{\left|x_{\text {new }}^{*}(i)\right|+e p s}\right\} \\
& \text { if } r \text { err }<0.5 * r e r r_{\text {tol }} \\
& \quad \text { sscal }=2 ; \text { accept }=1 \\
& \text { elseif } \text { rerr }<\text { rerr } r_{\text {tol }} \\
& \quad \text { sscal }=1 ; \text { accept }=1 \\
& \text { elseif } \text { rerr }<2 * \text { rerr } \text { tol } \\
& \quad \text { hscal }=0.5 ; \text { accept }=1 \\
& \text { else } \\
& \quad \text { hscal }=0.5 ; \text { accept }=0 \\
& \text { end } \\
& x_{\text {old }}=(1-\text { accept }) x_{\text {old }}+\text { accept } * x_{\text {new }}^{*} \\
& t=t+\text { accept } * \Delta t ; \Delta t=\max \left(\Delta t * h s c a l, \Delta t_{\text {min }}\right) \\
& \text { loop }
\end{aligned}
$$

where ode $(\cdot)$ represents the numerical solver of the ODEs and eps represents the floating-point relative accuracy in MATLAB. The relative error tolerance rerr $_{\text {tol }}$ is $10^{-4}$ in all simulations in this study. Other symbols in Eq. (D1) should be self-explanatory. 
Author contributions. J. Y. Tang developed the theory, conducted the analyses, and co-wrote the paper. W. J. Riley discussed the analyses and co-wrote the paper.

Acknowledgements. This research was supported by the Director, Office of Science, Office of Biological and Environmental Research of the US Department of Energy under contract no. DEAC02-05CH11231 as part of the Next-Generation Ecosystem Experiments (NGEE-Arctic) and the Accelerated Climate Model for Energy project in the Earth System Modeling program. We thank Niall Broekhuizen and an anonymous reviewer for their constructive comments, which improved the paper significantly.

Edited by: U. Seibt

\section{References}

Arora, V. K., Boer, G. J., Friedlingstein, P., Eby, M., Jones, C. D., Christian, J. R., Bonan, G., Bopp, L., Brovkin, V., Cadule, P., Hajima, T., Ilyina, T., Lindsay, K., Tjiputra, J. F., and Wu, T.: Carbon-Concentration and Carbon-Climate Feedbacks in CMIP5 Earth System Models, J. Climate, 26, 5289-5314, 2013.

Batstone, D. J., Keller, J., Angelidaki, I., Kalyuzhnyi, S. V., Pavlostathis, S. G., Rozzi, A., Sanders, W. T. M., Siegrist, H., and Vavilin, V. A.: The IWA Anaerobic Digestion Model No 1 (ADM1), Water Sci. Technol., 45, 65-73, 2002.

Bolley, C. and Crouzeix, M.: Conservation de la positivite lors de la discretization des problemes d'evolution parabolique, R. A. I. R. O. Numer. Anal., 12, 237-245, 1978.

Bouskill, N. J., Tang, J.Y., Riley, W. J., and Brodie, E. L.: Traitbased representation of biological nitrification: model development testing, and predicted community composition, Front. Microbiol., 3, doi:10.3389/fmicb.2012.00364, 2012.

Bouskill, N. J., Riley, W. J., and Tang, J. Y.: Meta-analysis of highlatitude nitrogen-addition and warming studies implies ecological mechanisms overlooked by land models, Biogeosciences, 11, 6969-6983, doi:10.5194/bg-11-6969-2014, 2014.

Broekhuizen, N., Rickard, G. J., Bruggeman, J., and Meister, A.: An improved and generalized second order, unconditionally positive, mass conserving integration scheme for biochemical systems, Appl. Numer. Math., 58, 319-340, 2008.

Chapin, F. S., Moilanen, L., and Kielland, K.: Preferential Use of Organic Nitrogen for Growth by a Nonmycorrhizal Arctic Sedge, Nature, 361, 150-153, 1993.

Fang, Y., Huang, M., Liu, C., Li, H., and Leung, L. R.: A generic biogeochemical module for Earth system models: Next Generation BioGeoChemical Module (NGBGC), version 1.0, Geosci. Model Dev., 6, 1977-1988, doi:10.5194/gmd-6-19772013, 2013.

Gerber, S., Hedin, L. O., Oppenheimer, M., Pacala, S. W., and Shevliakova, E.: Nitrogen cycling and feedbacks in a global dynamic land model, Global Biogeochem. Cy., 24, doi:10.1029/2008GB003336, 2010.

Hurrell, J. W., Holland, M. M., Gent, P. R., Ghan, S., Kay, J. E., Kushner, P. J., Lamarque, J. F., Large, W. G., Lawrence, D., Lindsay, K., Lipscomb, W. H., Long, M. C., Mahowald, N., Marsh,
D. R., Neale, R. B., Rasch, P., Vavrus, S., Vertenstein, M., Bader, D., Collins, W. D., Hack, J. J., Kiehl, J., and Marshall, S.: The Community Earth System Model A Framework for Collaborative Research, B Am. Meteorol. Soc., 94, 1339-1360, 2013.

Manzoni, S., Porporato, A., and Schimel, J. P.: Soil heterogeneity in lumped mineralization-immobilization models, Soil Biol. Biochem., 40, 1137-1148, 2008.

Moro, H., Kunito, T., Saito, T., Yaguchi, N., and Sato, T.: Soil microorganisms are less susceptible than crop plants to potassium deficiency, Arch. Agron. Soil Sci., 60, 1807-1813, 2014.

Oleson, K., Lawrence, D. M., Bonan, G. B., Drewniak, B., Huang, M., Koven, C. D., Levis, S., Li, F., Riley, W. J., Subin, Z. M., Swenson, S., Thornton, P. E., Bozbiyik, A., Fisher, R., Heald, C. L., Kluzek, E., Lamarque, J.-F., Lawrence, P. J., Leung, L. R., Lipscomb,W., Muszala, S. P., Ricciuto, D. M., Sacks,W. J., Sun, Y., Tang, J., and Yang, Z.-L.: Technical description of version 4.5 of the Community Land Model (CLM), NCAR Technical Note NCAR/TN-503+STR, 420 pp., doi:10.5065/D6RR1W7M, 2013.

Parton, W. J., Stewart, J. W. B., and Cole, C. V.: Dynamics of C, N, P and S in Grassland Soils - a Model, Biogeochemistry, 5, 109-131, 1988.

Reichert, P., Borchardt, D., Henze, M., Rauch, W., Shanahan, P., Somlyody, L., and Vanrolleghem, P.: River water quality model no. 1 (RWQM1): II. Biochemical process equations, Water Sci. Technol., 43, 11-30, 2001.

Riley, W. J., Subin, Z. M., Lawrence, D. M., Swenson, S. C., Torn, M. S., Meng, L., Mahowald, N. M., and Hess, P.: Barriers to predicting changes in global terrestrial methane fluxes: analyses using CLM4Me, a methane biogeochemistry model integrated in CESM, Biogeosciences, 8, 1925-1953, doi:10.5194/bg-8-19252011, 2011.

Russell: Practical wastewater treatment, John Wiley \& Sons, Inc. ISBN: 978-471-78044-1, 2006.

Sandu, A.: Positive numerical integration methods for chemical kinetic systems, J. Comput. Phys., 170, 589-602, 2001.

Schmidt, F., De Bona, F. D., and Monteiro, F. A.: Sulfur limitation increases nitrate and amino acid pools in tropical forages, Crop. Pasture Sci., 64, 51-60, 2013.

Tang, J. Y., Riley, W. J., Koven, C. D., and Subin, Z. M.: CLM4BeTR, a generic biogeochemical transport and reaction module for CLM4: model development, evaluation, and application, Geosci. Model Dev., 6, 127-140, doi:10.5194/gmd-6-127-2013, 2013.

Thomas, R. Q., Brookshire, E. N. J., and Gerber, S.: Nitrogen limitation on land: how can it occur in Earth system models?, Glob. Change Biol., 21, 1777-1793, 2015.

Thornton, P. E., Lamarque, J. F., Rosenbloom, N. A., and Mahowald, N. M.: Influence of carbon-nitrogen cycle coupling on land model response to $\mathrm{CO}_{2}$ fertilization and climate variability, Glob. Biogeochem Cy., 21, doi:10.1029/2006GB002868, 2007.

Vitousek, P. M. and Howarth, R. W.: Nitrogen Limitation on Land and in the Sea - How Can It Occur, Biogeochemistry, 13, 87115, 1991.

Vitousek, P. M., Porder, S., Houlton, B. Z., and Chadwick, O. A.: Terrestrial phosphorus limitation: mechanisms, implications, and nitrogen-phosphorus interactions, Ecol. Appl., 20, 5-15, 2010. 
Wang, Y. P., Law, R. M., and Pak, B.: A global model of carbon, nitrogen and phosphorus cycles for the terrestrial biosphere, Biogeosciences, 7, 2261-2282, doi:10.5194/bg-7-2261-2010, 2010.

Wieder, W. R., Cleveland, C. C., Smith, W. K., and Todd-Brown, K.: Future productivity and carbon storage limited by terrestrial nutrient availability, Nat. Geosci., 8, 441-444, 2015

Yang, X., Thornton, P. E., Ricciuto, D. M., and Post, W. M.: The role of phosphorus dynamics in tropical forests - a modeling study using CLM-CNP, Biogeosciences, 11, 1667-1681, doi:10.5194/bg-11-1667-2014, 2014.
Zhu, Q., Riley, W. J., Tang, J., and Koven, C. D.: Multiple soil nutrient competition between plants, microbes, and mineral surfaces: model development, parameterization, and example applications in several tropical forests, Biogeosciences, 13, 341-363, doi:10.5194/bg-13-341-2016, 2016. 